\title{
CAN HEMATOLOGICAL AND BIOCHEMICAL PARAMETERS FASTEN THE DIAGNOSIS OF COVID-19 IN EMERGENCY DEPARTMENTS?
}

\author{
Burak Katipoğlu ${ }^{1}$, Leyla Öztürk Sönmez ${ }^{2,3}{ }^{\oplus}$, Hülya Vatansev ${ }^{4}{ }^{\infty}$, Nalan Yüce $^{1}{ }^{\infty}$, \\ Mustafa Sabak ${ }^{5} \odot$, Lukasz Szarpak ${ }^{6,7,8} \odot$, Togay Evrin ${ }^{1} \odot$ \\ ${ }^{1}$ Department of Emergency Medicine, Ufuk University Medical Faculty, Ankara, Turkey \\ ${ }^{2}$ Department of Emergency Medicine, Beyhekim State Hospital, Konya, Turkey \\ ${ }^{3}$ Department of Physiology, Selcuklu Medical Faculty, Selcuk University, Konya, Turkey \\ ${ }^{4}$ Department of Chest Diseases, Meram Medical Faculty, Necmettin Erbakan University, Konya, Turkey \\ ${ }^{5}$ Department of Emergency Medicine, Nizip State Hospital, Gaziantep, Turkey \\ ${ }^{6}$ Department of Emergency Medicine, Maria Sklodowska-Curie Medical Academy, Warsaw, Poland \\ ${ }^{7}$ Department of Emergency Medicine, Polish Society of Disaster Medicine, Warsaw, Poland \\ ${ }^{8}$ Department of Emergency Medicine, Comprehensive Cancer Center in Bialystok, Poland
}

\begin{abstract}
INTRODUCTION: The primary aim of the study was to compare the laboratory and radiological parameters of COVID-19 positive and negative patients confirmed by Real-Time Reverse Transcription Polymerase Chain Reaction (RT-PCR), and Chest Computed Tomography (CCT) of patients admitting with the suspicion of COVID-19. The secondary purpose of the study was to find objective parameters to speed up the clinician for further examination, treatment or referral decision in COVID-19 suspicion.
\end{abstract}

MATERIAL AND METHODS: A total of 61 COVID-19 suspected patients were evaluated in the study. Swab samples were taken for RT-PCR analysis. CCT was taken for 42 patients who described dyspnea.

According to CCT and RT-PCR results, the patient population was divided into 2 groups as COVID-19 positive group $(n=32)$; and COVID-19 negative group $(n=29)$. Between two groups; demographic, clinical, laboratory and radiological parameters were compared.

RESULTS: Male gender $(p=0.03)$, PLR value $(p=0.021)$ and CO-RADS scores were higher in the COVID-19 positive group. Oxygen saturation $\left(\mathrm{SaO}_{2}\right)(p=0.027)$ and PCT, WBC, Neutrophil count, Lymphocyte count values were significantly low in COVID-19 positive group $(p=0.03, p=0.001, p=0.017, p=0.021$, respectively). PLR showed a positive correlation with fever, CRP, neutrophil count and NLR, which are indicators of inflammation.

CONCLUSIONS: $\mathrm{SaO}_{2}$, WBC, lymphocyte count, neutrophil count and low PCT levels, and PLR elevation showed a significant difference in COVID-19 patients in our retrospective cohort study examining the Turkish population. We believe that these results will allow clinicians to make quick decisions in patient management more simply.

KEY WORDS: CBC, COVID-19, CO-RADS, PLR, RT-PCR

Disaster Emerg Med J 2020; 5(4) 


\section{INTRODUCTION}

The disease that was caused by SARS-CoV-2 (COVID-19) first appeared in Wuhan, China in December 2019, then affected the whole world, and was recognized as pandemic by the World Health Organization (WHO) on February 11, 2020 [1].

COVID-19 is a very contagious disease and usually manifests with nonspecific respiratory symptoms like fever, cough, and shortness of breath [2]. The clinical manifestation of the disease usually occurs as fever (body temperature $37-38^{\circ} \mathrm{C}$ ), cough, nasal congestion and fatigue, which usually begin in 1 week in symptomatic patients [3], and can then progress to pneumonia [4].

Although it was shown that COVID-19 first occurred as a respiratory infection, the data that emerged later suggested that it should be considered as a systemic disease involving many systems, including cardiovascular, respiratory, gastrointestinal, neurological, hematopoietic and the immune system itself $[5,6]$. Since there are nonspecific symptoms and rapid deterioration of the clinical manifestations, the importance of accessible laboratory tests has increased to enable us to obtain quick results in the diagnosis of COVID-19 allowing us to determine its prognosis. Hematological and biochemical biomarkers provide us both objective perspective during the course of the disease and help to classify the disease as mild, severe or critical, allowing the diagnosis and treatment process to proceed faster [7].

For this reason, there are many studies that evaluate the relation of laboratory parameters with COVID-19, and these studies are usually associated with the clinical progression of COVID-19 [7].

The primary purpose of the present study was to compare the laboratory and radiological parameters of COVID-19 positive and negative patients confirmed by Real-Time Reverse Transcription Polymerase Chain Reaction (RT-PCR), and Chest Computed Tomography (CCT) of patients admitting with the suspicion of COVID-19. The secondary purpose of the study was to find objective parameters to speed up the clinician for further examination, treatment or referral decision in COVID-19 suspicion.

\section{MATERIAL AND METHODS}

Before the study was initiated, approval was obtained from the Ethics Board of Ufuk University (No: 20200521/9). A total of 61 COVID-19 suspected patients according to the Turkish Republic Ministry of Health COVID-19 Guideline, admitting to the university hospital emergency department between 05.03.2020 and 27.04.2020 were evaluated in the study [8]. According to the anamnesis of the patients; the demographic data, comorbidities, and smoking habits were recorded as the admission complaints. Detailed physical examinations were made after the vital findings of the patients were recorded. The Complete Blood Count (CBC), (including White Blood Cell (WBC), lymphocyte count, neutrophil count, platelet (PLT), Mean Platelet Volume $(\mathrm{MPV})$, plateletcrit $(\mathrm{PCT})$, Platelet Distribution Width (PDW), which are infection-related parameters in suspected patients of COVID-19, lactate dehydrogenase (LDH) from biochemical markers, C-reactive protein (CRP) and ferritin from acute phase reactants, and D-Dimer tests were performed for thrombosis. Also, Neutrophil/Lymphocyte Ratio (NLR) and Platelet-Lymphocyte Ratio (PLR) were calculated. Swab samples were taken for RT-PCR analysis. CCT was carried out for 42 patients who described dyspnea. The CCTs were classified by a radiologist according to CO-RADS 1-6 [9]. As previously defined, CORADS 1-3 groups were included in the Radiological COVID19 negative group; and CO-RADS 4-6 were included in the COVID-19 positive group.

According to CCT and RT-PCR results, the patient population was divided into 2 groups as COVID-19 positive patients COVID-19 positive group $(n=32)$; and according to CCT and RT-PCR results, COVID-19 negative patients COVID-19 negative group $(\mathrm{n}=29)$.

Exclusion criteria: The patients who were diagnosed with other pulmonary infections other than COVID-19, those with malignancies, those under the age of 18, and pregnant women were not included in the study.

The demographic, clinical, laboratory and radiological parameters between COVID-19 positive and negative groups were compared.

\section{Statistical Analysis}

Statistical analysis was performed with SPSS, v.23.0 statistical software (SPSS, Inc., Chicago, IL, USA). The categorical variables were described as frequencies and percentages. Continuous variables were presented as mean and standard deviations. Chi-square ( 2) tests were used to evaluate the relationship between categorical variables of study subgroups. Independent T test and Mann Whitney $U$ test for the comparison of two groups were the 
tests used in continuous variables. Pearson's correlation coefficients of continuous variables were also calculated. P values below 0.05 were considered statistically significant.

\section{RESULTS}

The mean age of the patients was $46.6 \pm 18.6$, and the male/female ratio was $44.3 \% / 55.7 \%(27 / 34)$. A total of $45.9 \%$ (28/61) patients did not have comorbidities, ut $27.9 \%$ (17/61) had multiple comorbidities, and among the comorbidities, the mostly detected ones were; hypertension: $37.7 \%$ (23/61), diabetes mellitus: $13.1 \%(8 / 61)$, coronary artery disease: $11.4 \%$ (7/61), asthma: $8.2 \%(5 / 61)$, respectively. A total of $29.3 \%$ patients had a smoking habit. Multiple admission complaints were determined in $41 \%$ patients (25/61), and the most common complaints were cough, fever and shortness of breath, respectively. CCT was performed because of clinical suspicion in $68.9 \%(42 / 61)$ patients. According to CCT results, in COVID-19 Reporting and Data System (CORADS) classification, the following results were obtained: CORADS1: $22(52.4 \%)$, CORADS 2:5 (11.9\%), CORADS 3: 2 (4.8\%), CORADS 4: 5 (11.9\%) CORADS 5: 1(2.4\%) CORADS6: 7(16.7\%). A total of 26 patients (42.6\%) were reported to be RT-PCR positive. Covid-19 was detected to be positive in 52.5\% (32/61) (CO-RADS 4-5-6 and/or RT-PCR+), and negative in 47.5\% (29/61) (CO-RADS 1-2-3 and RT-PCR).

The following were determined in the evaluation of the demographic, clinical, laboratory and radiological parameters of COVID-19 positive and negative groups. Male gender was more dominant in demographic parameters in COVID-19 positive group $(p=0.03)$; oxygen saturation $\left(\mathrm{SaO}_{2}\right)(p=0.027)$ from clinical parameters, and PCT, WBC, Neutrophil count, Lymphocyte count values were low at significant levels $(p=0.03, p=0.001, p=0.017$, $p=0.021$, respectively); PLR value was significantly higher $(p=0.021)$, and the CO-RADS scores from radiological parameters were higher as expected in COVID-19 positive group ( $p<0.001$ ) (Tab. 1).

In the correlation analysis: $\mathrm{SaO}_{2}$ showed a negative correlation with neutrophil count, NLR, WBC, CRP, ferritin, D-dimer and LDH. NLR showed a positive correlation with WBC, CRP and D-Dimer. PCT showed a negative correlation with $\mathrm{LDH}$ and positive correlation with CRP. PLR showed a positive correlation with fever, CRP, neutrophil count and NLR, which are indicators of inflammation. Details of the correlation analysis are shown in Table 2. Scatter Plots of laboratory parameters are shown in Figure 1.

\section{DISCUSSION}

It was shown in previous studies that although the first clinical symptom in detecting cases was pneumonia in COVID-19, it was also shown that it can also proceed with gastrointestinal symptoms or asymptomatically [10]. COVID-19 needs to be continuously improved and accelerated in terms of clinical diagnosis and treatment to control the spread, considering its spread and serious systemic effects $[10,11]$.

CCT is one of the most important means of diagnosis in many lung diseases, including pneumonia [12]. It was reported that the COVID-19 Reporting and Data System (CO-RADS) Classification for COVID-19 pneumonia is a categorical evaluation scheme for pulmonary involvement in unenhanced CCT, which has a predictive value in COVID-19 in patients with moderate and severe symptoms $[9,13]$. In our study, a total of 42 patients who described dyspnea complaints underwent CCT, and 13 patients $(30.9 \%)$ were reported to have findings associated with pneumonia.

In a study, it which COVID-19 positive 75 patients were evaluated, $60 \%$ of the patients were identified as male. In our study, male gender was found to be dominant in COVID-19 positive group, which is in line with the literature [14].

It was reported previously that the presence of comorbidity and smoking deteriorated the prognosis of COVID-19 patients [17]. In our study, $45.9 \%$ of patients had no comorbidities, and $29.3 \%$ of them were active smokers. However, no differences were detected between COVID-19 positive and negative groups in terms of smoking and comorbidity.

The most common complaints detected in COVID-19 patients were determined as fever, cough and sore throat [14]. In our study; however, the most common admission complaints were cough, fever and shortness of breath, respectively.

Huang et al. [16] referred to $\mathrm{SaO}_{2}$ as another indicator of the disease progression. It was reported previously that $\mathrm{SaO}_{2}$ was in close relation with hospitalization and mortality, and the cut-off value for $\mathrm{SaO}_{2}$ was 93\% in determining COVID-19 seriousness. In our study, $\mathrm{SaO}_{2}$ value was $94.06 \%$ in the COVID-19 positive group, and $95.9 \%$ in the COVID-19 negative group, and this difference was found to be 


\begin{tabular}{|c|c|c|c|c|}
\hline Parameters & Total $(n=61)$ & COVID-19+ $(n=32)$ & COVID-19- $(n=29)$ & $\mathrm{p}$ \\
\hline \multicolumn{5}{|c|}{ Demographics } \\
\hline Age & $46.6 \pm 18.6$ & $44.25 \pm 21.25$ & $49.24 \pm 15.13$ & 0.45 \\
\hline $\begin{array}{l}\text { Sex ( men/women) } \\
n(\%)\end{array}$ & $\begin{array}{c}27 / 34 \\
(44.3 / 55.7)\end{array}$ & $20 / 12(62.5 / 37.5)$ & $\begin{array}{c}7 / 22 \\
(24.1 / 75.9)\end{array}$ & 0.003 \\
\hline Comorbidity n (\%) & $33(54.1)$ & $16(50)$ & $17(56.6)$ & 0.5 \\
\hline $\begin{array}{l}\text { Smoking }(+) \\
\mathrm{n}(\%)\end{array}$ & $24(39.3)$ & $11(34.4)$ & $13(44.8)$ & 0.44 \\
\hline \multicolumn{5}{|l|}{ Clinical Parameters } \\
\hline $\mathrm{SaO}_{2}(\%)$ & $94.84 \pm 7.4$ & $94.06 \pm 8.2$ & $95.9 \pm 6.3$ & 0.027 \\
\hline Fever (CO) & $36.79 \pm 0.64$ & $36.9 \pm 0.64$ & $36.6 \pm 0.62$ & 0.143 \\
\hline \multicolumn{5}{|l|}{ Laboratory Parameters } \\
\hline WBC $\left(10^{3} / \mu \mathrm{L}\right)$ & $8.06 \pm 4.27$ & $6.90 \pm 4.72$ & $9.34 \pm 3.36$ & 0.001 \\
\hline Neutrophil $\left(10^{3} / \mu \mathrm{L}\right)$ & $5.52 \pm 3.9$ & $5.01 \pm 4.37$ & $6.08 \pm 3.37$ & 0.017 \\
\hline Lymphocyte $\left(10^{3} / \mu \mathrm{L}\right)$ & $2.06 \pm 1.1$ & $1.79 \pm 0.96$ & $2.37 \pm 1.18$ & 0.021 \\
\hline NLR & $4.67 \pm 7.3$ & $5.23 \pm 8.86$ & $4.06 \pm 5.21$ & 0.977 \\
\hline $\mathrm{CRP}(\mathrm{mg} / \mathrm{L})$ & $26.93 \pm 55.1$ & $29.09 \pm 56.29$ & $24.55 \pm 54.77$ & 0.322 \\
\hline D-DIMER $\mu \mathrm{g} / \mathrm{L}$ & $326.77 \pm 575.6$ & $304.40 \pm 299.93$ & $351.44 \pm 780.56$ & 0.056 \\
\hline FERRITIN $\mu \mathrm{g} / \mathrm{L}$ & $213.75 \pm 378$ & $278.96 \pm 481.75$ & $141.79 \pm 197.81$ & 0.549 \\
\hline $\mathrm{LDH} U / \mathrm{L}$ & $205.49 \pm 137$ & $219.28 \pm 178.84$ & $190.27 \pm 68.61$ & 0.301 \\
\hline 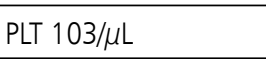 & $257.50 \pm 68.9$ & $250.93 \pm 71.10$ & $265.03 \pm 68.05$ & 0.52 \\
\hline MPV fL & $7.58 \pm 1.34$ & $7.36 \pm 1.07$ & $7.83 \pm 1.58$ & 0.37 \\
\hline PCT (\%) & $0.19 \pm 0.04$ & $0.18 \pm 0.04$ & $0.20 \pm 0.04$ & 0.03 \\
\hline PDW (10(GSD)) & $20.29 \pm 1.14$ & $20.23 \pm 0.95$ & $20.35 \pm 1.34$ & 0.913 \\
\hline PLR & $165.49 \pm 126.39$ & $187.64 \pm 136.4$ & $141.06 \pm 111.48$ & 0.021 \\
\hline \multicolumn{5}{|c|}{ Radiological Parameters } \\
\hline $\begin{array}{l}\text { CORADS n (\%) } \\
1 \\
2 \\
3 \\
4 \\
5 \\
6\end{array}$ & $\begin{array}{c}22(52.4) \\
5(11.9) \\
2(4.8) \\
5(11.9) \\
1(2.4) \\
7(16.7)\end{array}$ & $\begin{array}{c}0 \\
0 \\
0 \\
5(38.5) \\
1(7.7) \\
7(53.8)\end{array}$ & $\begin{array}{c}22(75.9) \\
5(17.2) \\
2(6.9) \\
0 \\
0 \\
0\end{array}$ & $<0.001$ \\
\hline
\end{tabular}

LDH — lactate dehydrogenase PLT; MPV — Mean Platelet Volume; NLR — Neutrophi/Lymphocyte Ratio CRP; PCT — plateletcrit; PDW — Platelet Distribution Width; PLR — Platelet-Lymphocyte Ratio; $\mathrm{SaO}_{2}-$ oxygen saturation; $\mathrm{WBC}-$ White Blood Cell

significant $(p=0.027)$. We believe that the low $\mathrm{SaO}_{2}$ levels in our patient group were because of the damage the virus did directly on the lungs.

Yang et al. [17] classified COVID-19 patients as severe/non-severe. WBC was found to be higher at significant levels in the severe group, and a significant correlation was detected between NLR elevation and COVID-19 risk. Terpos et al. [18] reported that the WBC count was normal or slightly lower in the early stages of the disease. Similarly, in a study investigating laboratory parameters in the diagnosis of COVID-19 in the first 2 months of the outbreak, WBC was detected to be decreased in $33.7 \%$ of patients [3]. In our study, on the other hand, the WBC count was found to be low in the COVID-19 positive group. This decrease can be associated with the decrease in neutrophil and lymphocyte parameters, but according to the data we obtained, we believe that it does not provide adequate data in COVID-19 suspicion.

It was shown in different studies that COVID-19 positive patients had decreased lymphocyte count, 


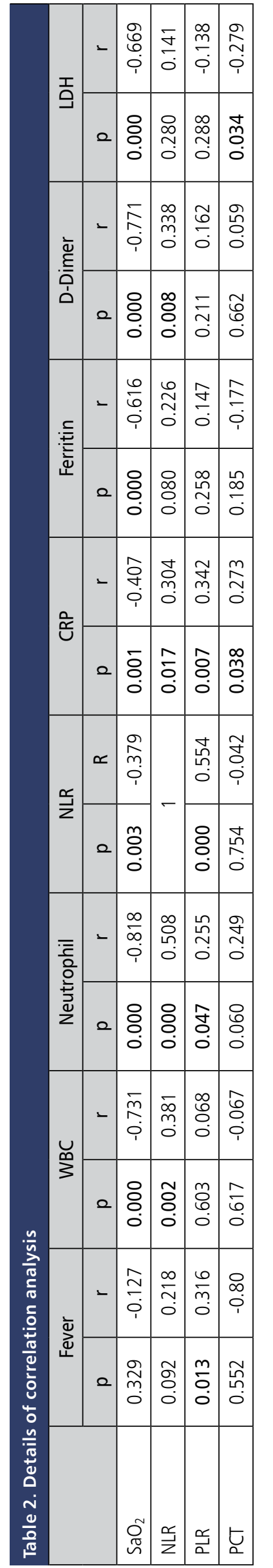

which was associated with the prognosis $[14,17]$. It was determined that this was because of the lithic effect of SARS-COV-2 in the cells and the atrophy effect of cytokine activation on lymphoid organs after SARS-COV-2 binds to angiotensin-converting enzyme 2 (ACE2) receptors expressed on the lymphocyte surface, which altogether caused a decrease in the lymphocyte turnover rate $[19,20]$. In our study, the lymphocyte count was measured to be significantly low in the COVID-19 positive group $(p=0.021)$.

Neutrophil count was found to be elevated in severe COVID-19 group in various studies $[14,17]$. In a prospective study that included 416 patients, $19.7 \%$ of the patients had myocardial damage, and these patients had a higher number of neutrophils than others [21]. It was considered that these results were associated with the septic condition and advanced organ damage $[17,21]$. Contrary to these data, the number of neutrophils was found to be lower in COVID-19 positive group in our study $(p=0.017)$. We believe that this is related to the fact that the patients included in our study were in good condition, and were patients in the early stages of the disease.

It was reported that the NLR level is high in severe COVID-19 group, which was associated with in-hospital mortality $[14,15]$. In our study; however, no differences were detected in terms of NLR in COVID-19 positive group compared to the negative group. The reason for the difference between previous studies might be methodological, or can be explained with the fact that no significant elevation was detected in neutrophil count in our study.

In a previous study examining the relations between PLT parameters and mortality, it was determined that thrombocytopenia and low PCT were associated with an increase in in-hospital mortality; however, increases were detected in the PLT values in surviving patients during the disease, and low PCT was identified as an independent risk factor for mortality [22]. In our study, although the PLT count and MPV values were below the reference values in COVID-19 positive group, no significant differences were detected in this regard. It was found that the PCT value was lower in the COVID-19 positive group, which, as a finding, also has the potential to show that the COVID-19 positive patient group had a higher risk than the COVID-19 negative group ( $p=0.03$ ).

PLR is a new index that shows inflammation. It was shown previously that PLR can be used as 

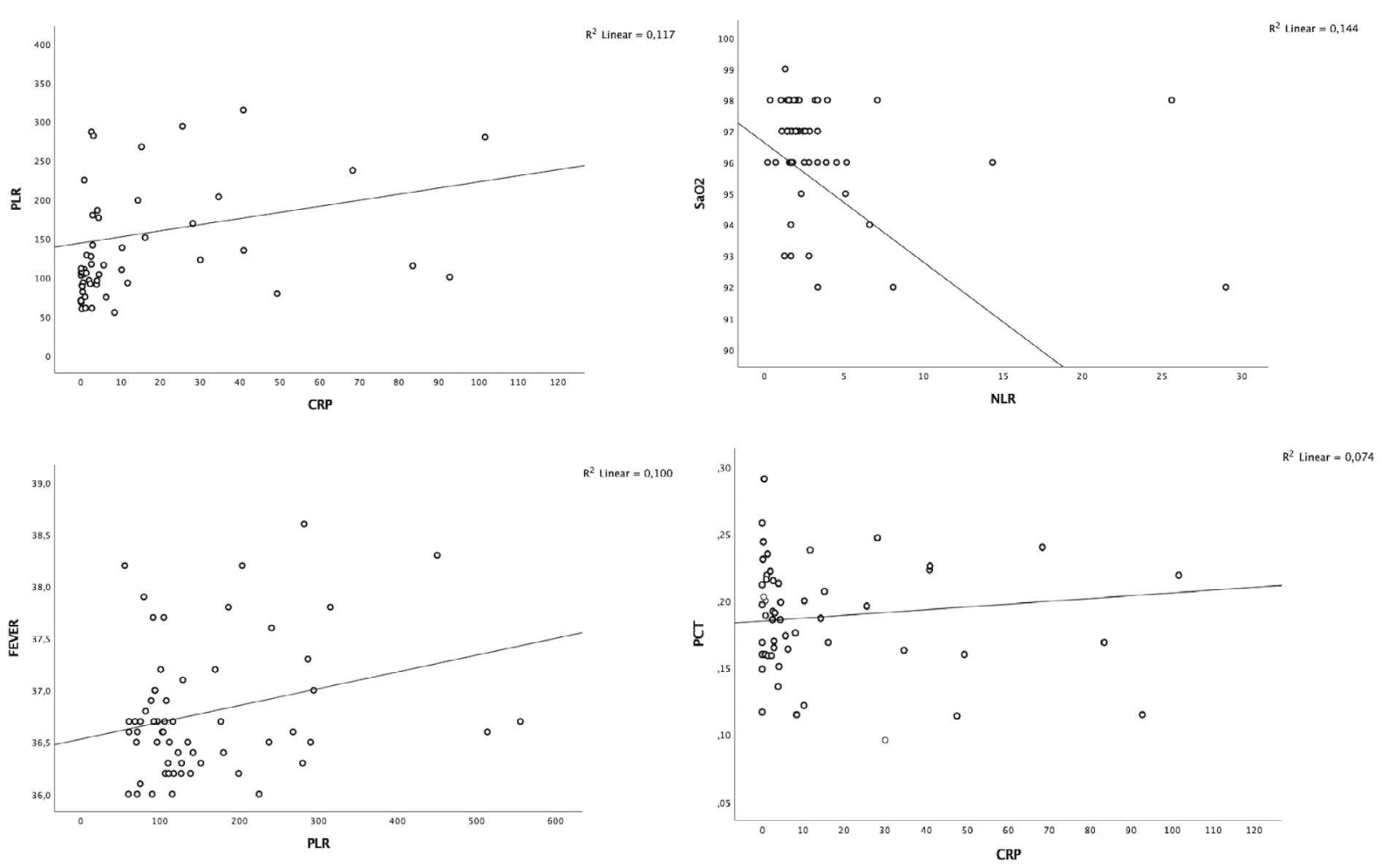

FIGURE 1. Scatter plots of laboratory parameters

the potential inflammatory indicator for clinical diagnosis of community-based pneumonia [23]. Qu et al. [23] showed the relation of low lymphocyte levels in admission with prognosis. Also, low PLT levels were associated with more serious diseases, and elevated PLR showed the degree of cytokine storm in monitoring COVID-19 patients. In our study, PLR value was higher in COVID-19 positive patients $(p=0.021)$; and in the correlation analysis, it was shown that PLR had a positive correlation with inflammation indicators, fever, CRP, neutrophil count and NLR. The significantly high PLR levels in COVID-19 positive patient group in our study may provide the clinician with an idea on early diagnosis. According to the results of our study, we believe that PLR might have an important value in COVID-19 suspected patients in terms of the severity of inflammation, it may be supportive in the decision for tomography in patients admitted to emergency department with suspicion of COVID-19, and may be an important indicator in the hospitalization decision of COVID-19 positive patients.

It was determined in our study that the lymphocyte count, neutrophil count, WBC and PCT were low in COVID-19 positive patients; and PLR and CO-
RADS were determined to be high. These parameters were associated with disease severity in previous studies. Depending on the density in the laboratory work, the hemogram result can be obtained in approximately 5 minutes, and RT-PCR result must be expected after at least 1-3 hours. Rapid identification and isolation of people suspected of SARSCoV-2 infection are essential [24]. In this context, we believe that these parameters, especially the PLR elevation, which supports the presence of inflammation, will provide important clues to the clinician for hospitalization, timely treatment and early isolation of patients admitted to the emergency department with non-specific symptoms and findings.

\section{CONCLUSION}

The $\mathrm{SaO}_{2}, \mathrm{WBC}$, lymphocyte count, neutrophil count and low PCT levels, and PLR elevation showed a significant difference in COVID-19 patients in our retrospective cohort study examining the Turkish population. We believe that these results will allow clinicians to make quick decisions in patient management more simply; however, more studies are needed with wider patient populations. 
Ethics: Consent according to Helsinki declaration was taken from Ufuk University Faculty of Medicine ethics committee before the study

(No: 20200521/9).

Statement of: The authors declared no potential conflicts of interest with respect to the research, authorship, and/or publication of this article.

Source of Funding: None.

\section{REFERENCES}

1. Ge H, Wang $X$, Yuan $X$, et al. The epidemiology and clinical information about COVID-19. Eur J Clin Microbiol Infect Dis. 2020; 39(6): 1011-1019, doi: 10.1007/s10096-020-03874-z, indexed in Pubmed: 32291542.

2. Wang W, Tang J, Wei F. Updated understanding of the outbreak of 2019 novel coronavirus (2019-nCoV) in Wuhan, China. J Med Virol. 2020; 92(4): 441-447, doi: 10.1002/jmv.25689, indexed in Pubmed: 31994742.

3. Guan WJ, Ni ZY, Hu Yu, et al. China Medical Treatment Expert Group for Covid-19. Clinical Characteristics of Coronavirus Disease 2019 in China. N Engl J Med. 2020; 382(18): 1708-1720, doi: 10.1056/ NEJMoa2002032, indexed in Pubmed: 32109013.

4. Çınar E. Türkdoğan Pratik Acil Tıp, Section 10: Pneumonia, 1st Ed. (Istanbul: EMA Medicine Publisher. ; 2020: pp.

5. Mehta P, McAuley DF, Brown M, et al. HLH Across Speciality Collaboration, UK. COVID-19: consider cytokine storm syndromes and immunosuppression. Lancet. 2020; 395(10229): 1033-1034, doi: 10.1016/S0140-6736(20)30628-0, indexed in Pubmed: 32192578.

6. Bangash MN, Patel J, Parekh D. COVID-19 and the liver: little cause for concern. Lancet Gastroenterol Hepatol. 2020; 5(6): 529-530, doi: 10.1016/S2468-1253(20)30084-4, indexed in Pubmed: 32203680.

7. Kermali M, Khalsa RK, Pillai $K$, et al. The role of biomarkers in diagnosis of COVID-19 - A systematic review. Life Sci. 2020; 254: 117788, doi: 10.1016/j.lfs.2020.117788, indexed in Pubmed: 32475810.

8. Turkish Health Ministry SARS Cov- 2 Infection Guide, English version avaible at: https://hsgm. saglik gov tr/depo/birimler/goc_sagligi/ covid19/rehber/COVID-19_Rehberi20200414_eng_v4_002_14 05. ; 2020: pdf.

9. Prokop $M$, van Everdingen W, van Rees Vellinga T, et al. COVID-19 Standardized Reporting Working Group of the Dutch Radiological Society. CO-RADS: A Categorical CT Assessment Scheme for Patients Suspected of Having COVID-19-Definition and Evaluation. Radiology. 2020; 296(2): E97-E9E104, doi: 10.1148/radiol.2020201473, indexed in Pubmed: 32339082.

10. Chan JFW, Yuan S, Kok KH, et al. A familial cluster of pneumonia associated with the 2019 novel coronavirus indicating person-to-person transmission: a study of a family cluster. Lancet. 2020; 395(10223): 514-523, doi: 10.1016/S0140-6736(20)30154-9, indexed in Pubmed: 31986261.
11. Zhao S, Lin $Q$, Ran J, et al. Preliminary estimation of the basic reproduction number of novel coronavirus (2019-nCoV) in China, from 2019 to 2020: A data-driven analysis in the early phase of the outbreak. Int J Infect Dis. 2020; 92: 214-217, doi: 10.1016/j.ijid.2020.01.050, indexed in Pubmed: 32007643.

12. Bronchogenic cyst. Definitions. 2020, doi: $10.32388 / 3$ nvus9.

13. Radiological Society of North America Expert Consensus Statement on Reporting Chest CT Findings Related to COVID-19. Endorsed by the Society of Thoracic Radiology, the American College of Radiology, and RSNA. Radiology: Cardiothoracic Imaging. 2020, doi: 10.1148/ ryct.2020200152. podcast.

14. Fu J, Kong J, Wang W, et al. The clinical implication of dynamic neutrophil to lymphocyte ratio and D-dimer in COVID-19: A retrospective study in Suzhou China. Thromb Res. 2020; 192: 3-8, doi: 10.1016/j. thromres.2020.05.006, indexed in Pubmed: 32407937.

15. Liu Y, Du X, Chen J, et al. Neutrophil-to-lymphocyte ratio as an independent risk factor for mortality in hospitalized patients with COVID-19. J Infect. 2020; 81(1): e6-ee12, doi: 10.1016/j.jinf.2020.04.002, indexed in Pubmed: 32283162.

16. Huang $C$, Wang $Y$, LiX, et al. Clinical features of patients infected with 2019 novel coronavirus in Wuhan, China. Lancet. 2020; 395(10223): 497-506, doi: 10.1016/S0140-6736(20)30183-5, indexed in Pubmed: 31986264.

17. Yang AP, Liu JP, Tao WQ, et al. The diagnostic and predictive role of NLR, d-NLR and PLR in COVID-19 patients. Int Immunopharmacol. 2020; 84: 106504, doi: 10.1016/j.intimp.2020.106504, indexed in Pubmed: 32304994.

18. Terpos E, Ntanasis-Stathopoulos I, Elalamy I, et al. Hematological findings and complications of COVID-19. Am J Hematol. 2020; 95(7): 834-847, doi: 10.1002/ajh.25829, indexed in Pubmed: 32282949.

19. Xu H, Zhong L, Deng J, et al. High expression of ACE2 receptor of 2019nCoV on the epithelial cells of oral mucosa. Int J Oral Sci. 2020; 12(1): 8, doi: 10.1038/s41368-020-0074-x, indexed in Pubmed: 32094336.

20. Chan JW, Zhang A, Yuan S, et al. Simulation of the Clinical and Pathological Manifestations of Coronavirus Disease 2019 (COVID-19) in a Golden Syrian Hamster Model: Implications for Disease Pathogenesis and Transmissibility. Clinical Infectious Diseases. 2020, doi: 10.1093/cid/ciaa325.

21. Shi S, Qin Mu, Shen Bo, et al. Association of Cardiac Injury With Mortality in Hospitalized Patients With COVID-19 in Wuhan, China. JAMA Cardiol. 2020; 5(7): 802-810, doi: 10.1001/jamacardio.2020.0950, indexed in Pubmed: 32211816.

22. Liu Y, Sun W, Guo Y, et al. Association between platelet parameters and mortality in coronavirus disease 2019: Retrospective cohort study. Platelets. 2020; 31(4): 490-496, doi: 10.1080/09537104.2020.1754383, indexed in Pubmed: 32297540.

23. Qu R, Ling Y, Zhang $Y H$, et al. Platelet-to-lymphocyte ratio is associated with prognosis in patients with coronavirus disease-19. J Med Virol. 2020 [Epub ahead of print], doi: 10.1002/jmv.25767, indexed in Pubmed: 32181903.

24. Smereka J, Szarpak L, Filipiak K. Modern medicine in COVID-19 era. Disaster and Emergency Medicine Journal. 2020, doi: 10.5603/demj. a2020.0012. 